
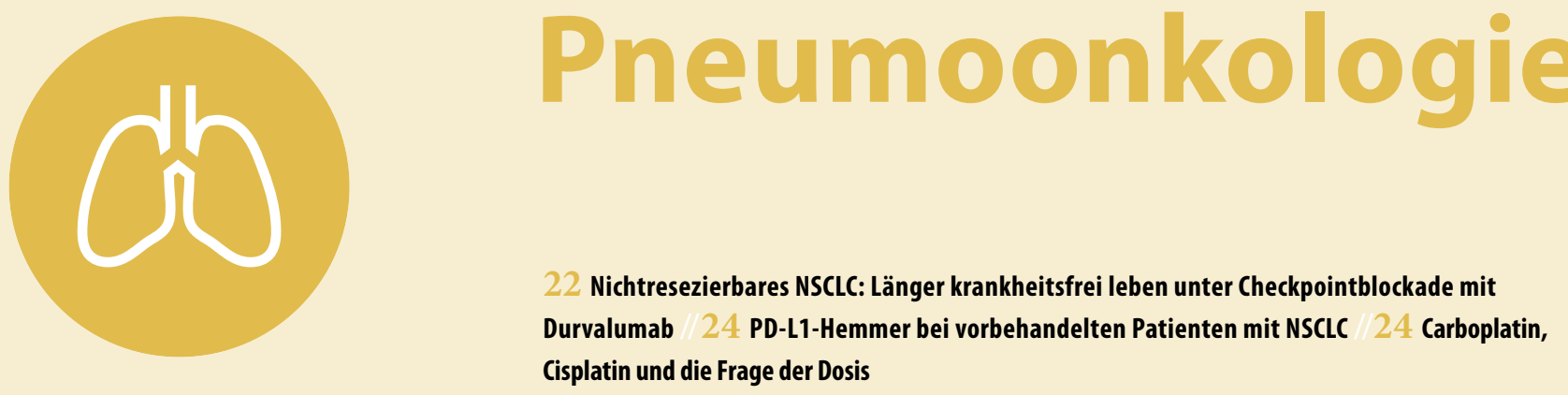

\section{Nichtresezierbares NSCLC: Länger krankheitsfrei leben unter Checkpointblockade mit Durvalumab}

Unter Therapie mit Durvalumab, einem Antikörper, der gegen PD-L1 („programmed cell death-ligand 1") gerichtet ist, war das krankheitsfreie Überleben von Patienten mit nichtresezierbarem nichtkleinzelligen Lungenkarzinom (NSCLC) länger als unter Placebo.

Nur ur bei wenigen Patienten mit lokal fortgeschrittenem, unresezierbarem NSCLC gelingt ein dauerhafter Stopp der Krankheitsprogression durch eine Chemoradiotherapie. Konsolidierungstherapien sind deshalb dringend nötig. In einer Phase-III-Studie hat ein internationales Forscherteam um Scott J. Antonia, Tampa, FL/USA, den immunmodulierenden Antikörper Durvalumab im Vergleich zu Placebo erprobt. Durvalumab blockiert den Liganden PD-L1, der verschiedene Immunantworten herunterreguliert.

Im Rahmen der Studie wurden Patienten im Verhältnis 2:1 auf 2 Behandlungsarme randomisiert. Sie erhielten entweder

_Durvalumab $(10 \mathrm{mg} / \mathrm{kg}$ Körperge-

wicht intravenös; q2w für bis zu 12 Monate) oder

Placebo (q2w für bis zu 12 Monate).

Durvalumab wurde an den Tagen 1 bis 42 nach Beenden der Chemoradiotherapie verabreicht. 473 Patienten erhielten eine Konsolidierungstherapie mit Durvalumab, 236 ein Placebo.

Das mediane progressionsfreie Überleben war unter Durvalumab 3-mal so lang wie unter Placebo (16,8 vs. 5,6 Monate; Hazard Ratio 0,52; $<<0,001)$. Die Rate für das progressionsfreie Überleben betrug nach 12 Monaten unter Durvalumab 55,9\% gegenüber $35,3 \%$ unter $\mathrm{Pla}$ cebo, nach 18 Monaten $44,2 \%$ gegenüber
27,0\%. Auch die Ansprechrate war unter Durvalumab signifikant höher als unter Placebo (28,4 vs. $16,0 \%$; $p<0,001)$, und die mittlere Dauer des Ansprechens war erheblich länger (72,8 vs. $46,8 \%$ der Patienten zeigten noch nach 18 Monaten ein Ansprechen). Die mittlere Zeit bis zum Tod oder bis zum Auftreten von Fernmetastasen war unter Durvalumab etwa 1,5-mal so lang wie unter Placebo ( 23,2 vs. 14,6 Monate; $\mathrm{p}<0,001$ ).

Nebenwirkungen vom Grad 3/4 traten in beiden Gruppen nahezu gleich häufig auf (29,9 vs. $26,1 \%)$. Die häufigste Toxizität vom Grad 3/4 war Pneumonie $(4,4$ vs. $3,8 \%)$. Allerdings unterbrachen mehr Patienten in der Durvalumab-Gruppe als in der Placebo-Gruppe die Einnahme der Studienmedikation aufgrund von Nebenwirkungen (15,4 vs. $9,8 \%)$.

Fazit: Die positiven Ergebnisse in einer unselektierten Patientenpopulation und unabhängig von einer PD-L1-Expression auf den Tumorzellen vor Behandlungsbeginn lassen ein profundes Potenzial von Durvalumab als adjuvante Therapie bei Patienten mit einem NSCLC im Stadium III nach Standardbehandlung vermuten.

Christian Behrend

Antonia SJ et al. Durvalumab after Chemoradiotherapy in Stage III Non-Small-Cell Lung Cancer. N Engl J Med. 2017;377(20):1919-29.

\section{Durvalumab: Deutsche Studie zur} Zweitlinientherapie beim NSCLC

Unter Federführung von Jonas Kuon von der Thoraxklinik am Uniklinikum Heidelberg wird zurzeit die Duration-Studie (AIO-YMO/TRK-0416; NCT03345810) vorbereitet: In dieser offenen Phase-II-Studie wird der Einsatz von Durvalumab bei älteren bzw. gebrechlichen Patienten mit nichtkleinzelligem Lungenkarzinom (NSCLC) untersucht. Die Patienten werden entweder behandelt mit: einer Standard Mono- bzw. Kombinationschemotherapie plus Durvalumab-Erhaltung oder mit einer Standard Mono- bzw. Kombinationschemotherapie allein

Aufgenommen werden Patienten, die älter als 70 Jahre sind, an einem metastasierten NSCLC ohne molekulare Alterationen von ALK bzw. EGFR leiden, noch nicht systemisch vorbehandelt wurden und für die eine Cisplatin-basierte Chemotherapie nicht infrage kommt. Endpunkte der Studie sind u. a.: Rate der therapieassoziierten Nebenwirkungen vom Grad 3/4 (primärer Endpunkt) progressionsfreies Überleben objektive Ansprechrate Gesamtüberleben

Gemäß https://clinicaltrials.gov ist der Studienstart für Dezember 2017 vorgesehen, das Ende der Datenerhebung bzgl. des primären Endpunkts für Juni 2022. Bei der Studie handelt es sich um eine Untersuchung, die unter dem Label der Young Medical Oncologists (YMO) der Arbeitsgemeinschaft für Internistische Onkologie (AIO) durchgeführt wird. Laut AIO-Website steht die Studie für weitere Zentren offen.

Zum Thema YMO lesen Sie mehr im Bericht zum AIO-Herbstkongress im selben Heft ab Seite 70 [Grätzel von Grätz P. Im Focus Onkologie. 2017;20(12):70-71].

Moritz Borchers 